This item was submitted to Loughborough's Research Repository by the author.

Items in Figshare are protected by copyright, with all rights reserved, unless otherwise indicated.

\title{
Monte Carlo simulation modelling of aircraft dispatch with known faults
}

PLEASE CITE THE PUBLISHED VERSION

PUBLISHER

(C) Institute of Electrical and Electronics Engineers (IEEE)

VERSION

VoR (Version of Record)

LICENCE

CC BY-NC-ND 4.0

REPOSITORY RECORD

Prescott, Darren R., and J.D. Andrews. 2009. "Monte Carlo Simulation Modelling of Aircraft Dispatch with Known Faults". figshare. https://hdl.handle.net/2134/5482. 
This item was submitted to Loughborough's Institutional Repository (https://dspace.lboro.ac.uk/) by the author and is made available under the following Creative Commons Licence conditions.

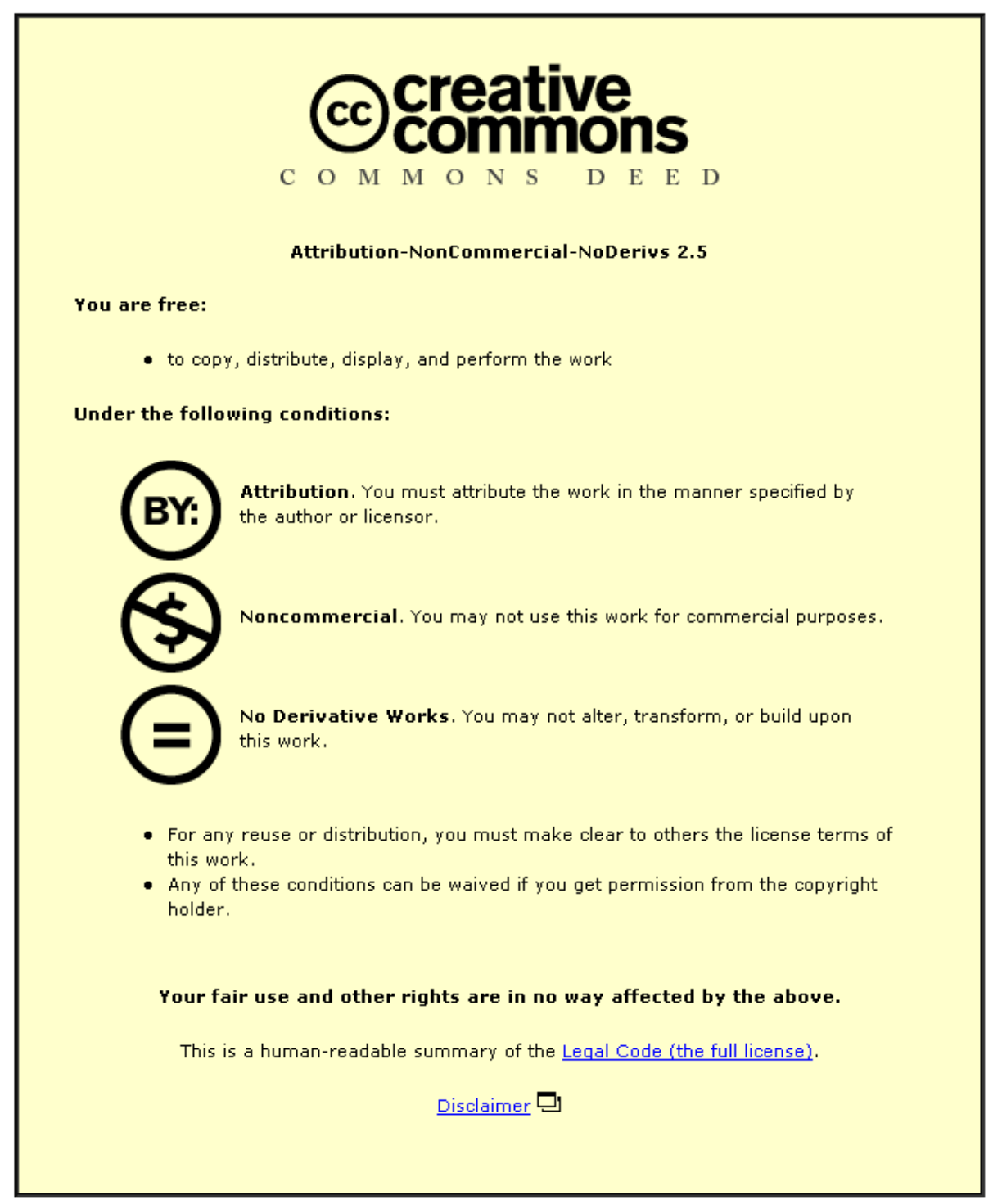

For the full text of this licence, please go to: http://creativecommons.org/licenses/by-nc-nd/2.5/ 


\title{
Monte Carlo Simulation Modelling of Aircraft Dispatch with Known Faults
}

\author{
Darren PRESCOTT, John ANDREWS \\ Department of Aeronautical and Automotive Engineering \\ Loughborough University \\ Loughborough, LE11 3TU, UK \\ E-mail: D.R.Prescott@lboro.ac.uk; J.D.Andrews@lboro.ac.uk
}

\begin{abstract}
Time-Limited Dispatch (TLD) allows usage of redundancy within aircraft engine control systems in order to dispatch aircraft with faults present in these systems for limited periods. Such degraded-redundancy dispatch enables aircraft operators to reduce delays and cancellations of aircraft flights through efficient maintenance scheduling. When TLD is applied certification requirements must be met, which ensure that system failure rates do not exceed specified levels. This paper presents a Monte Carlo simulation approach to obtaining the required system failure rates and outlines advantages of the approach in ensuring certification requirements are met.
\end{abstract}

Keywords- Monte Carlo simulation; Time-Limited Dispatch; degraded redundancy

\section{INTRODUCTION}

Time-Limited Dispatch (TLD) came into existence following the introduction of Full Authority Digital Engine Control (FADEC) systems to commercial aircraft in the mid1980s. FADEC systems are electronic engine control systems which regulate engine thrust from the beginning of fuel metering to the point of fuel shutoff. When introduced, FADEC systems replaced hydromechanical control (HMC) systems and it was to be the first time that pilots would have no access to a (HMC) system in the event of electronic system failure [1].

FADEC systems are based around a dual channel control system. They contain reliable electronic components and are designed in such a way that critical loops and functions are covered by a degree of redundancy. Despite expectations that these features would be positive, there was an initial negative impact on aircraft dispatch following the introduction of FADEC systems. This was due to the imposition of the dispatch criteria that were applied to HMC systems, which, it turned out, were overly restrictive. There was scope to take advantage of redundancies to reduce unwelcome flight delays and cancellations due to FADEC system faults. This was the basis of TLD, which was introduced to allow dispatch of aircraft with faults present in their FADEC systems for limited periods, after which the fault must be repaired in order for further dispatch to be allowed.

\section{A. Certification Requirements}

Before TLD may be applied to a FADEC system, there are a number of certification requirements that must be met. These relate to the levels of reliability demanded of the system, and in particular to Loss of Thrust Control (LOTC) rates related to the system [2]. The average LOTC rate of the system must not exceed 10 failures per $10^{6}$ flight hours, a level set to match that required of HMC systems prior to the introduction of FADEC systems. In addition to this there are restrictions placed on the instantaneous LOTC rate, which is calculated when the FADEC system is operating with faults present and must not exceed 100 failures per $10^{6}$ flight hours. There are four dispatch categories that relate to TLD faults:

- Do Not Dispatch (DND),

- $\quad$ Short Time Dispatch (STD),

- $\quad$ Long Time Dispatch (LTD),

- Manufacturer/Operator Defined Dispatch (MDD).

By definition, DND faults prohibit aircraft dispatch when they are present and therefore any fault states from which the instantaneous LOTC rate exceeds 100 failures per $10^{6}$ flight hours must be categorised as DND. The other dispatch categories have associated dispatch intervals, measured in aircraft flight hours. When a fault that is related to one of these dispatch categories occurs, the aircraft may be dispatched with that fault for up to the length of the associated dispatch interval before it must be cleared from the system. The instantaneous failure rate to LOTC when dispatching with LTD and STD faults must not exceed 75 and 100 failures per $10^{6}$ flight hours respectively. MDD faults either do not fall into any of the other categories or do not affect the system LOTC rates. For any faults, more restrictive dispatch categories than those determined using the instantaneous LOTC rates may be applied.

\section{B. Maintenance Strategies}




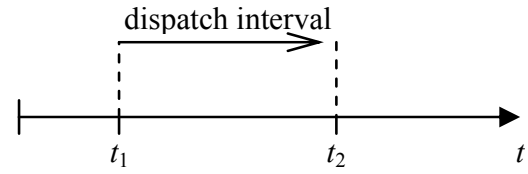

Figure 1. MEL maintenance.

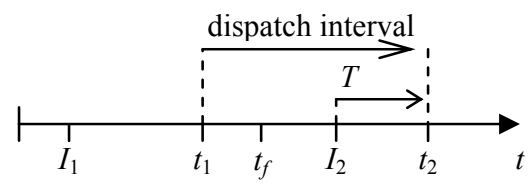

Figure 2. PIR maintenance.

There are two maintenance strategies that can be used when TLD is applied, Minimum Equipment List (MEL) maintenance and Periodic Inspection and Repair (PIR) maintenance. MEL maintenance is a time-since-fault repair strategy that may be used when system faults are revealed, i.e. the time of occurrence of the fault is known. Figure 1 illustrates MEL maintenance, where a fault occurs at time $t_{1}$, at which time a dispatch interval is initiated and the fault must be cleared from the system by $t_{2}$, at the end of the dispatch interval. PIR maintenance, Figure 2, involves periodically inspecting the system for faults. When a fault occurring at $\mathrm{tf}$ is discovered at an inspection the time that it occurred will not be known. It is assumed to have occurred at the midpoint $\left(t_{1}\right)$ of the interval between the current inspection $\left(I_{2}\right)$ and the previous one $\left(I_{1}\right)$ and the dispatch interval is initiated at this point. The inspection interval may be adjusted to allow dispatch for a certain period $T$ after the inspection and the fault must be cleared by time $t_{2}$.

\section{Multiple Faults}

When more than one fault is present in the system it is possible to take advantage of the application of TLD to allow flexible repair strategies at maintenance. This could, for example, allow an optimisation of the number of maintenance operations that must take place, or allow aircraft dispatch until appropriate parts are available. In order to illustrate some of the options, two examples of multiple fault occurrences relating to MEL maintenance are now presented. Figure 3 depicts the occurrence of two faults, $A$ and $B$, with dispatch intervals ending at $t_{1}$ and $t_{2}$ respectively. Upon reaching time $t_{1}$ fault $\mathrm{A}$ must be cleared from the system. Fault $B$ could also be cleared, allowing dispatch until further faults occur or fault $B$ may be left in the system, allowing further dispatch until time $t_{2}$, when it must be cleared from the system. Figure 4 shows a similar scenario, where the presence of $A$ or $B$ alone leads to the application of a LTD interval (ending at $t_{1}$ for $A$ and $t_{2}$ for $B$ ). In this case the simultaneous presence of both $A$ and $B$ within the system leads to an increase in instantaneous LOTC rate such that a STD interval (ending at $t_{3}$ ) must be applied. At time $t_{3}$ there are a number of maintenance options. $A$ alone may be cleared, leaving $B$ in the system and thus allowing dispatch until $t_{2}$, when $A$ must be cleared. $B$ alone may be cleared, leaving $\mathrm{A}$ in the system and allowing dispatch until $t_{1}$, when $B$ must be cleared. The final alternative is that both faults are cleared from the system.

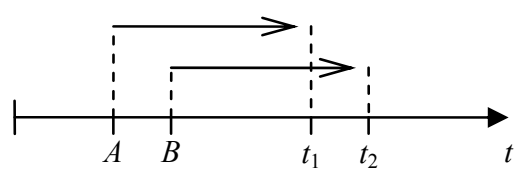

Figure 3. Multiple faults (MEL maintenance).

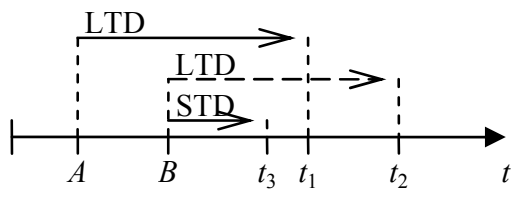

Figure 4. The combination of multiple faults (MEL maintenance).

\section{MODELling TLD}

Before applying TLD to a system it is necessary to demonstrate that the certification requirements for the average and instantaneous LOTC rates can be met using a suitable reliability analysis technique. Dispatch criteria (TLD categories for faults) must be assigned to all dispatchable faults and the length of dispatch intervals must also be set. Standard models such as the kinetic tree theory of Fault Tree analysis [3] or Markov analysis of conventional Markov models are unsuitable; the former due to the dependencies introduced by TLD and its associated maintenance and the latter due to the problem of state space explosion.

\section{A. Recommended Models}

There are two recommended approaches to modelling TLD [4] and computing the average LOTC rate for a system given dispatch criteria (dispatch categories for faults) and lengths of the dispatch intervals. The first is a time-weighted averages (TWA) approach, which looks to compute the LOTC rate by averaging the LOTC rates of the system from each of its dispatchable configurations. The second is a simple Markov model containing a reduced number of system states, which overcomes problems associated with a state space explosion and means analysis is possible. Both of these models consider only full-up system states (with no faults present) and dispatchable (STD and LTD) TLD fault states. However, both of these models require the calculation of two sets of failure rates: failure rates into the dispatchable system fault states and failure rates to LOTC from the dispatchable system fault states (on which the system dispatch criteria are dependent). The calculation of these failure rates is not necessarily a straightforward task, and it is this calculation that may ultimately hamper any attempt to gain accurate results from these models. Due to the limited space available in this paper the recommended models are not discussed in greater detail here, but further details can be found in references [4-8]. References [5-8] show some applications of the models to simple systems. Figure 5 shows the required model inputs, further inputs that must be calculated and also the model output.

\section{B. Proposed Monte Carlo Simulation Model}

Previous work has shown that Monte Carlo simulation is well-suited to modelling TLD [5-8]. It is a flexible modelling approach that can easily handle dependencies introduced by 
TLD, the different possible maintenance strategies and ordering of faults. An outline of a Monte Carlo simulation algorithm for calculating the LOTC rate of a system is given in Figure 6. The basic premise is that a number of system lifetimes are modelled and TLD faults and maintenance of the instantaneous failure rates to LOTC from each of the dispatchable system fault states. Different system lifetimes may be modelled, as can different flight times. MEL or PIR maintenance can be modelled, either exclusively (for example, both STD and LTD faults covered by MEL maintenance) or in

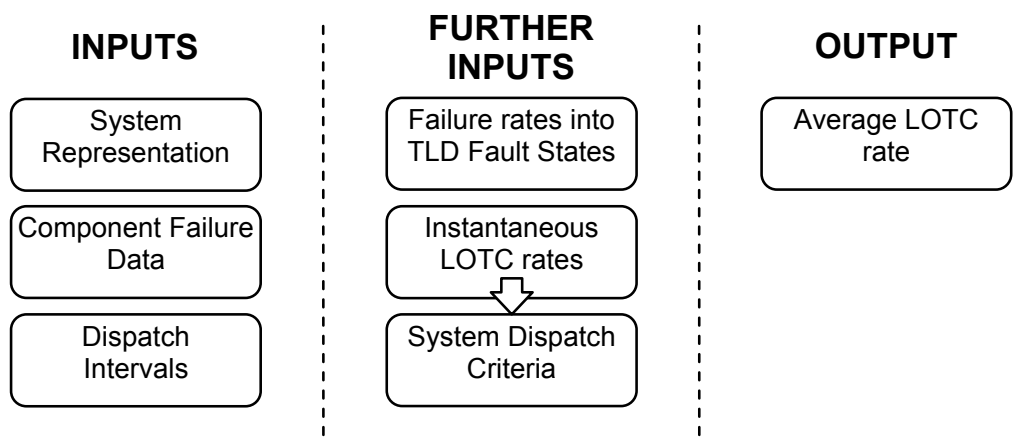

Figure 5. Recommended model inputs and outputs.

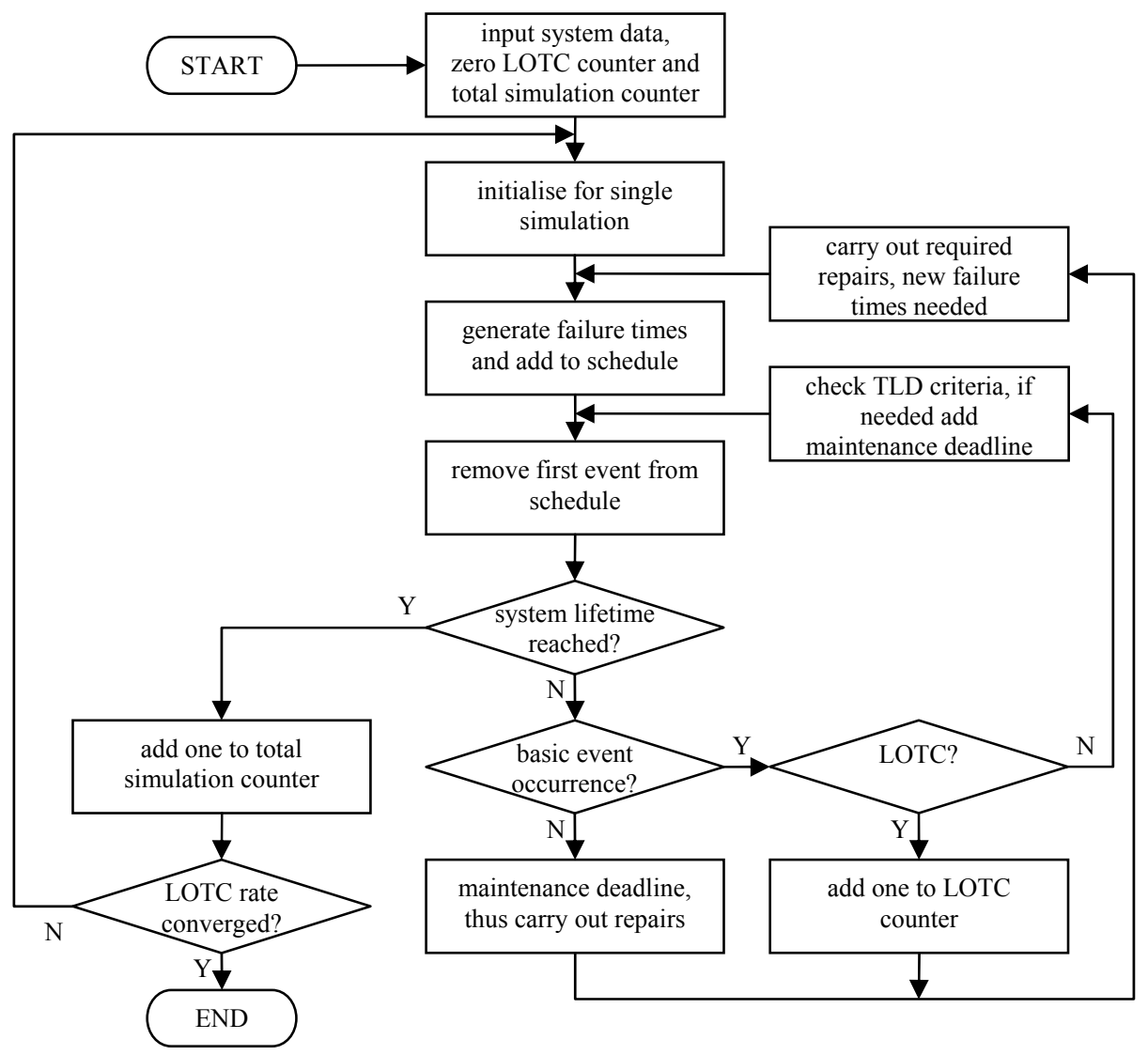

Figure 6. Overview of an algorithm for finding the system LOTC rate.

those faults are considered. System component failure distributions are sampled to provide the times to failure during single simulations and the dispatch intervals related with TLD categories are used to determine for how long the system can be dispatched with specific faults present. A record is kept of the number of LOTC events that occur and the total system lifetime that is modelled. This allows the calculation of a LOTC rate for the system. A computer code has been developed that incorporates this basic algorithm. The developed Monte Carlo code also has the capability to compute combination (such as LTD faults covered by PIR maintenance and STD faults covered by MEL maintenance). The code also allows consideration of different repair strategies at maintenance, such as those outlined when considering multiple faults in Section $I-C$. This degree of flexibility is not offered by the recommended approaches.

An iterative procedure has also been developed, which takes advantages of the possibility to compute instantaneous LOTC rates from the individual fault states and allows the 
dispatch criteria to be set accordingly. It works by initially assuming that all TLD faults will be treated as LTD faults. The system is then modelled and any of the instantaneous LOTC rates for these faults that do not satisfy the certification aircraft. The approach does not require calculations to be made prior to TLD modelling, which is in contrast to the recommended approaches, which require the calculation of failure rates into the TLD fault states and out of them to the
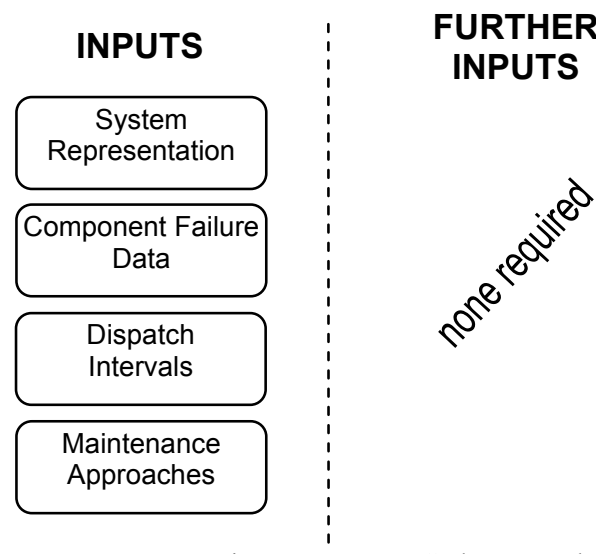

\section{OUTPUTS}

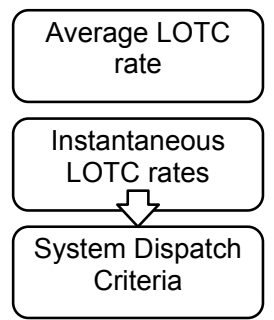

requirements are selected and the dispatch category set to the appropriate fault category according to the instantaneous LOTC rate observed. Another set of simulations are then run. The process sets lower order fault categories first, then works up through higher order fault categories and continues until all TLD faults have instantaneous LOTC rates that satisfy the certification requirements of the dispatch category applied to them. In this way it is possible to model the application of TLD to a system and set the dispatch criteria for that system whilst ensuring that all certification requirements relating to average and instantaneous LOTC rates are met. Figure 7 shows the required model inputs and outputs available when using the Monte Carlo simulation approach.

\section{COMPARISON OF THE TWO MODELLING APPROACHES}

Comparing Figures 5 and 7 gives a feel for how the recommended approaches differ from the developed Monte Carlo approach. Considering the inputs, both use a system representation and component failure data. The recommended models require this data to be used to calculate further inputs: the failure rates into the TLD fault states and the LOTC rates from these fault states. This is not necessarily a simple task. The Monte Carlo approach does not need this - the system representation and component failure data are used within simulations to check for LOTC events and produce component failure times respectively. The instantaneous LOTC rates are produced as an output in the MCS approach. Note how, because of this, it is possible to produce the system dispatch criteria as outputs whereas they are inputs to the recommended models. Both approaches also require the length of the dispatch intervals (for STD and LTD faults) to be defined. In addition the Monte Carlo approach can take other inputs, such as the maintenance approaches used, which is shown in Figure 7 and other quantities such as system lifetime and flight length.

\section{CONCLUSIONS}

Presented in this paper is a brief overview of a Monte Carlo simulation approach to modelling the application of TLD to
LOTC state. This therefore gives the Monte Carlo approach an advantage in that it offers an integrated approach to modelling TLD, specifying dispatch criteria that satisfy certification requirements and modelling different maintenance strategies. However, the recommended approaches to modelling TLD do offer some benefits. They are very simple models that can be easily implemented and in the instance where real failure data can be gathered from a system the models can be used to obtain the average system LOTC rate. The advantage for the Monte Carlo approach would come when real data is not available. The Monte Carlo approach also offers advantages in terms of being able to model different repair strategies.

\section{REFERENCES}

[1] H. Larsen, and G. Horan, "Time-Limited Dispatch: An Interactive Training and Self-Study Course," Keybridge Technologies, Inc,2002.

[2] FAA Memorandum, Policy for Time-Limited Dispatch (TLD) of Engines Fitted with Full Authority Digital Engine Control Systems, Policy No. ANE-1993-33.28TLD-R1, 2001.

[3] W.E. Vesely, "A Time Dependent Methodology for Fault Tree Evaluation," Nuclear Design and Engineering, 1970, No.13, pp.337-360.

[4] SAE ARP5107 Rev A, "Guidelines for Time-Limited Dispatch (TLD) Analysis for Electronic Engine Control Systems," SAE International, 2005 .

[5] D.R. Prescott, and J.D. Andrews,. "Aircraft Safety Modelling for TimeLimited Dispatch," Proceedings of the Annual Reliability and Maintainability Symposium, Virginia, USA (CD-ROM),2005.

[6] D.R. Prescott, and J.D. Andrews,. "A Comparison of Modelling Approaches for the Time-Limited Dispatch of Aircraft," IMechE Part O: Journal of Risk and Reliability, 2006, Vol.220, No.01, pp.9-20.

[7] D.R. Prescott, and J.D. Andrews, "Modelling and Specification of TimeLimited Dispatch Categories for Commercial Aircraft," ASME Journal of Dynamic Systems, Measurement and Control, 2008, Vol.130, Iss.2.

[8] D.R. Prescott, and J.D. Andrews, "The Safe Dispatch of Aircraft With Known Faults," International Journal of Performability Engineering, Condition Monitoring and Condition Based Maintenance Special Issue, Kumar, U., (ed), 2008, Vol.4, No.3, pp.243-253.

[9] G.B. Wang, H.Z. Huang, L.S. Sun, "A hybrid cross-entropy algorithm for reliability assessment of configuration-redundancy systems," Eksploatacja i Niezawodnosc - Maintenance and Reliability, 2009, No.3, pp.4-13. 